\title{
Geometry-Adapted Hexahedral Meshes Improve Accuracy of Finite-Element-Method-Based EEG Source Analysis
}

\author{
Carsten H. Wolters*, Alfred Anwander, Guntram Berti, and Ulrich Hartmann
}

\begin{abstract}
Mesh generation in finite-element- (FE) methodbased electroencephalography (EEG) source analysis generally influences greatly the accuracy of the results. It is thus important to determine a meshing strategy well adopted to achieve both acceptable accuracy for potential distributions and reasonable computation times and memory usage. In this paper, we propose to achieve this goal by smoothing regular hexahedral finite elements at material interfaces using a node-shift approach. We first present the underlying theory for two different techniques for modeling a current dipole in FE volume conductors, a subtraction and a direct potential method. We then evaluate regular and smoothed elements in a four-layer sphere model for both potential approaches and compare their accuracy. We finally compute and visualize potential distributions for a tangentially and a radially oriented source in the somatosensory cortex in regular and geometry-adapted three-compartment hexahedra FE volume conductor models of the human head using both the subtraction and the direct potential method. On the average, node-shifting reduces both topography and magnitude errors by more than a factor of 2 for tangential and $\mathbf{1 . 5}$ for radial sources for both potential approaches. Nevertheless, node-shifting has to be carried out with caution for sources located within or close to irregular hexahedra, because especially for the subtraction method extreme deformations might lead to larger overall errors. With regard to realistic volume conductor modeling, node-shifted hexahedra should thus be used for the skin and skull compartments while we would not recommend deforming elements at the grey and white matter surfaces.
\end{abstract}

Index Terms-Dipole, direct potential approach, EEG, finite-element method, geometry-adapted hexahedra, realistic head modeling, regular hexahedra, source reconstruction, subtraction potential approach.

\section{INTRODUCTION}

$\mathbf{T}$ HE localization of current sources in the human brain from surface electroencephalography (EEG) measurements (the inverse problem) requires a model for the forward problem, i.e.,

Manuscript received June 23, 2006; revised November 5, 2006. This work was supported by the IST-program of the European Community, project SIMBIO (http://www.simbio.de), by the Deutsche Forschungsgemeinschaft (WO1425/1-1) and the NIH NCRR Center for Integrative Biomedical Computing (http://www.sci.utah.edu/cibc). Asterisk indicates corresponding author.

${ }^{*} \mathrm{C}$. H. Wolters is with the Institute for Biomagnetism and Biosignalanalysis, Westfälische Wilhelms-Universität Münster, Malmedyweg 15, 48149 Münster, Germany, and also with the Scientific Computing and Imaging Institute, University of Utah, Salt Lake City, UT 84112 USA (e-mail: carsten.wolters@ unimuenster.de).

A. Anwander is with the Max Planck Institute for Human Cognitive and Brain Sciences, 04103 Leipzig, Germany (e-mail: anwander@ cbs.mpg.de).

G. Berti is with the C\&C Research Laboratories, NEC Europe Ltd., 53757 St. Augustin, Germany (e-mail: berti@ccrl-nece.de).

U. Hartmann is with the University of Applied Sciences Koblenz, Department of Mathematics, RheinAhrCampus Remagen, 53424 Remagen, Germany (e-mail: Hartmann@RheinAhrCampus.de).

Color versions of one or more of the figures in this paper are available online at http://ieeexplore.ieee.org.

Digital Object Identifier 10.1109/TBME.2007.890736 the determination of surface potentials from current sources in the volume. Because of its ability to treat volume conductors of arbitrary complexity and model inhomogeneous and anisotropic tissue conductivity, the finite-element method (FEM) has become popular to solve the forward problem [1], [2], [4], [5], [14], [19], [25]-[27]. An essential prerequisite for FE modeling is the generation of a mesh which represents the geometric and electric properties of the volume conductor. So far, surfacebased tetrahedral tesselations were mainly used [1], [2], [4], [5], [14], [25], [27]. Only few studies examined regular hexahedral elements exploiting the spatial discretization inherent in medical tomographic data [19], [23], whose excellent performance has been shown in a recent accuracy study [19], and found to perform better than the surface-based tetrahedra [23]. Adaptive methods [2], [4] disallow use of lead field bases [7], [8], [21], [24] (discussed later) and loose efficiency when solving the inverse problem. The problematic stair-like approximation of curved boundaries with regular hexahedra has been addressed by [6] in a biomechanical context, where it was shown that a node-shifting approach can significantly reduce errors in von Mises stress at the surface, in spite of detrimental effects of deformed elements.

In this paper, we first present the underlying theory for two different techniques for modeling a current dipole in FE volume conductors, a subtraction and a direct potential method. We then test the hypothesis that node-shift hexahedra surface smoothing reduces EEG forward modeling errors. We evaluate the new mesh-generation approach in a four-layer sphere model for both the subtraction and the direct potential method, using statistical metrics for a comparison of the numerical results with an analytical solution at surface measurement points. We then present electric potential visualization results for a tangentially and a radially oriented source in the somatosensory cortex in regular and geometry-adapted three-compartment hexahedra FE volume conductor models of the human head using both the subtraction and the direct potential method. We finally discuss our results and conclude in the last chapter.

\section{METHODS}

\section{A. The FEM-Based EEG Forward Problem}

In the quasistatic approximation of Maxwell's equations, the distribution of electric potentials $\Phi$ in the head domain $\Omega$ of conductivity $\sigma$, resulting from a primary current $\mathbf{j}^{p}$ is governed by the Poisson equation with homogeneous Neumann boundary conditions on the head surface $\Gamma=\partial \Omega[18]$

$$
\nabla \cdot(\sigma \nabla \Phi)=\nabla \cdot \mathbf{j}^{p}=J^{p} \text { in } \Omega, \quad\langle\sigma \nabla \Phi, \mathbf{n}\rangle=0 \text { on } \Gamma
$$


with $\mathbf{n}$ the unit surface normal, and a reference electrode with given potential, i.e., $\Phi\left(\mathbf{x}_{\text {ref }}\right)=0$.

The primary currents are generally modeled by a mathematical dipole at position $\mathbf{x}_{0} \in \mathbb{R}^{3}$ with the moment $\mathbf{M}_{0} \in \mathbb{R}^{3}[18]$,

$$
\mathbf{j}^{p}(\mathbf{x})=\mathbf{M}_{0} \delta\left(\mathbf{x}-\mathbf{x}_{0}\right) .
$$

1) The Subtraction Approach: For the subtraction method [1], [2], [4], [14], [19], [23], the total potential $\Phi$ is split into two parts

$$
\Phi=\Phi^{\infty}+\Phi^{\text {corr }}
$$

where the singularity potential $\Phi^{\infty}$ is defined as the solution for a dipole in an unbounded homogeneous conductor with constant conductivity $\sigma^{\infty}$ (the conductivity at the source position). The solution of Poisson's equation for the singularity potential

$$
\Delta \Phi^{\infty}=\frac{\nabla \cdot \mathbf{j}^{p}}{\sigma^{\infty}}
$$

can be formed analytically by use of (2)[18]

$$
\Phi^{\infty}(\mathbf{x})=\frac{1}{4 \pi \sigma^{\infty}} \frac{\left\langle\mathbf{M}_{0},\left(\mathbf{x}-\mathbf{x}_{0}\right)\right\rangle}{\left|\mathbf{x}-\mathbf{x}_{0}\right|^{3}} .
$$

Subtracting (4) from (1) yields a Poisson equation for the correction potential

$$
-\nabla \cdot\left(\sigma \nabla \Phi^{\mathrm{corr}}\right)=\nabla \cdot\left(\left(\sigma-\sigma^{\infty}\right) \nabla \Phi^{\infty}\right) \text { in } \Omega
$$

with inhomogeneous Neumann boundary conditions at the surface

$$
\left\langle\sigma \nabla \Phi^{\mathrm{corr}}, \mathbf{n}\right\rangle=-\left\langle\sigma \nabla \Phi^{\infty}, \mathbf{n}\right\rangle \text { on } \Gamma \text {. }
$$

The advantage of (5) is that the right-hand side is free of any source singularity, because in a subdomain around the dipole, the conductivity $\sigma-\sigma^{\infty}$ is zero. For the numerical approximation of the correction potential, we use the FE method with isoparametric transformations of the deformed cube elements to the reference cube element and piecewise trilinear basis functions $\varphi_{i}$ at nodes $\boldsymbol{\xi}_{i}$, i.e., $\varphi_{i}(\boldsymbol{\xi})_{i}=1$ and $\varphi_{j}\left(\boldsymbol{\xi}_{i}\right)=0$ for all $j \neq i$. When projecting both the singularity and the correction potential into the FE space, i.e., $\Phi^{\infty}(\mathbf{x}) \approx \Phi_{h}^{\infty}(\mathbf{x})=\sum_{i=1}^{N} \varphi_{i}(\mathbf{x}) u_{i}^{\infty}$ with $u_{i}^{\infty}=\Phi^{\infty}\left(\boldsymbol{\xi}_{i}\right)$ and $\Phi^{\text {corr }}(\mathbf{x}) \approx \Phi_{h}^{\text {corr }}(\mathbf{x})=\sum_{j=1}^{N} \varphi_{j}(\mathbf{x}) u_{j}^{\text {corr }}$, and applying variational and $\mathrm{FE}$ techniques to (5),(6), we finally arrive at a linear system

$$
K \underline{u}^{\mathrm{corr}}=\underline{J}^{\mathrm{corr}}
$$

with the stiffness matrix

$$
K^{[i, j]}=\int_{\Omega}\left\langle\sigma \nabla \varphi_{i}, \nabla \varphi_{j}\right\rangle
$$

the right-hand side vector

$$
\underline{J}^{\mathrm{corr}}=-K^{\mathrm{corr}} \underline{u}^{\infty}-S \underline{u}^{\infty}
$$

with matrices

$$
\begin{aligned}
\left(K^{\mathrm{corr}}\right)^{[i, j]} & =\int_{\Omega}\left\langle\left(\sigma-\sigma^{\infty}\right) \nabla \varphi_{i}, \nabla \varphi_{j}\right\rangle, \\
S^{[i, j]} & =\int_{\Gamma}\left\langle\sigma^{\infty} \nabla \varphi_{j}, \mathbf{n}\right\rangle \varphi_{i}
\end{aligned}
$$

and with $\underline{u}^{\infty}=\left(u_{1}^{\infty}, \ldots, u_{N}^{\infty}\right)$ being the coefficient vector for $\Phi_{h}^{\infty}$. We then seek for the coefficient vector $\underline{u}^{\text {corr }}=\left(u_{1}^{\text {corr }}, \ldots, u_{N}^{\text {corr }}\right)$ and, using (3), the total potential can be computed. In a small subdomain around the dipole position, the linear approximation of the singularity potential $\Phi^{\infty}$ through $\Phi_{h}^{\infty}$ is quite rough, but $\sigma-\sigma^{\infty}$ is zero so that, under the condition that the source is not too close to a next conductivity jump, (5) and (6) are appropriately modeled with the presented linear FE approach.

2) Direct Potential Approach: Even if the mathematical dipole (2), consisting of an infinitesimal separation between the two poles, an infinite current sink and source and a finite dipole moment, is widely used in source analysis, a smoother model based on finite monopolar source and sink distributions and separations might be even more realistic [5], [19], [21], [26], [27]. However, from a more practical point of view, dipole vectors contain more information (strength and orientation) and ease the interpretation of inversely calculated source configurations. Therefore, it has been proposed to approximate the mathematical dipole with a smoother blurred dipole using a collection of monopolar sources and sinks on all neighboring FE mesh nodes in order to optimally match a given dipole moment vector [5]. In the following, we present the theory for the direct potential approach using the blurred dipole model. We will closely follow the ideas of [5], where the blurred dipole model was used in tetrahedra volume conductors, but our matrix-based reformulation easifies understanding and implementation and allows a direct comparison with the subtraction approach especially with regard to the computational effort in both tetrahedra and regular and node-shifted hexahedra FE volume conductors. Starting from the basic relation for a dipole moment $\mathbf{T}_{l} \in \mathbb{R}^{3}$ at position $\mathbf{x}_{l} \in \mathbb{R}^{3}$ ( $\mathbf{x}_{l}$ being an arbitrary position in the grey matter compartment, i.e., not necessarily an FE node), $\mathbf{T}_{l}=\int_{\Omega}\left(\mathbf{x}-\mathbf{x}_{l}\right) J^{p}(\mathbf{x}) d \mathbf{x}$ (see, e.g., [16, formula (2.92)]), and assuming discrete sources on only $C$ neighboring FE mesh nodes, it is $\mathbf{T}_{l}=\sum_{c=1}^{C} \Delta \mathbf{x}_{c l} \underline{j}_{l}^{[c]}$ with $\Delta \mathbf{x}_{c l}$ denoting the vector from FE node $c$ to source position $\mathbf{x}_{l}$. When using higher moments $\underline{\bar{T}}_{l}^{r} \in \mathbb{R}^{n_{0}+1}$ with $n_{0}=1,2$ and the Cartesian direction $r(r=x, y, z)$, it is

$$
\left(\underline{\bar{T}}_{l}^{r}\right)^{[n]}=\left(\underline{\bar{T}}_{l}^{r}\right)^{[n]}\left(\underline{j}_{l}\right)=\sum_{c=1}^{C}\left(\Delta \bar{x}_{c l}^{r}\right)^{n} \underline{j}_{l}^{[c]} \quad \forall n \in 0, \ldots, n_{0}
$$

(for a motivation of higher moments see [5]). The bar indicates a scaling with a reference length $a_{\text {ref }}$, so that

$$
\Delta \bar{x}_{c l}^{r}=\frac{\Delta x_{c l}^{r}}{a_{\text {ref }}} \stackrel{!}{<} 1
$$

is dimensionless and the physical dimension of the resultant scaled $n^{t h}$ order moment, $\left(\bar{T}_{l}^{r}\right)^{[n]}$, is that of a current (i.e., A, 
Ampère). $a_{\text {ref }}$ has to be chosen so that $\Delta \bar{x}_{c l}^{r}$ is smaller 1 . This is expressed by the exclamation mark in (11). The equation is well known from mechanical engineering, where small forces in combination with long lever arms have the same effect on the system as large forces in combination with short lever arms. If we now define the matrix $\bar{X}_{l}^{r} \in \mathbb{R}^{\left(n_{0}+1\right) \times C}$, the moment vector $\bar{M}_{l}^{r} \in \mathbb{R}^{n_{0}+1}$, computed from the given dipole moment vector $\overline{\mathbf{M}}_{l}$, and the diagonal source weighting matrix $\bar{W}_{l}^{r} \in \mathbb{R}^{C \times C}$ by

$$
\begin{aligned}
\left(\bar{X}_{l}^{r}\right)^{[n, c]} & =\left(\Delta \bar{x}_{c l}^{r}\right)^{n} \\
\left(\underline{\bar{M}}_{l}^{r}\right)^{[n]} & =M_{l}^{r}\left(\frac{1}{2 a_{\text {ref }}}\right)^{n}\left(1-(-1)^{n}\right) \\
\bar{W}_{l}^{r} & =\operatorname{DIAG}\left(\left(\Delta \bar{x}_{1 l}^{r}\right)^{s}, \ldots,\left(\Delta \bar{x}_{C l}^{r}\right)^{s}\right)
\end{aligned}
$$

with $s=0$ or $s=1$, then we compute the monopole load vector $j_{l}$ of the blurred dipole on the $C$ neighboring FE nodes from the given dipole moment vector $\mathbf{M}_{l}$ at position $\mathrm{x}_{l}$ by means of minimizing the following functional:

$$
\begin{aligned}
F_{\lambda}\left(\underline{j}_{l}\right) & =\left\|\underline{\bar{M}}_{l}^{r}-\underline{\bar{T}}_{l}^{r}\left(\underline{j}_{l}\right)\right\|_{2}^{2}+\lambda\left\|\bar{W}_{l}^{r} \underline{j}_{l}\right\|_{2}^{2} \\
& =\left\|\underline{\bar{M}}_{l}^{r}-\bar{X}_{l}^{r} \underline{j}_{l}\right\|_{2}^{2}+\lambda\left\|\bar{W}_{l}^{r} \underline{j}_{l}\right\|_{2}^{2} \\
& \stackrel{!}{=} \min .
\end{aligned}
$$

The first part of the functional $F_{\lambda}$ ensures a minimal difference between the moments of the blurred dipole $\bar{T}_{l}^{r}$ and the target ones $\underline{M}_{l}^{r}$, while the second part, a Tikhonov-Phillips regularizer with $\lambda$ the dipole regularization parameter, smoothes the monopole distribution in a weighted sense and enables a unique minimum for $F_{\lambda}$. The solution of the minimization problem is given by

$$
\left(\left(\bar{X}_{l}^{r}\right)^{t r} \bar{X}_{l}^{r}+\lambda\left(\bar{W}_{l}^{r}\right)^{t r} \bar{W}_{l}^{r}\right) \underline{j}_{l}=\left(\bar{X}_{l}^{r}\right)^{t r} \underline{\bar{M}}_{l}^{r}
$$

(see, e.g., [13, Theorem 4.2.1]) so that the final solution for the monopole source vector $\underline{j}_{l}$ of the blurred dipole is given by

$$
\begin{aligned}
\underline{j}_{l}=\left(\sum_{r=1}^{3}\left\{\left(\bar{X}_{l}^{r}\right)^{t r} \bar{X}_{l}^{r}+\lambda\left(\bar{W}_{l}^{r}\right)^{t r} \bar{W}_{l}^{r}\right\}\right)^{-1} & \\
& \times \sum_{r=1}^{3}\left\{\left(\bar{X}_{l}^{r}\right)^{t r} \underline{M}_{l}^{r}\right\} .
\end{aligned}
$$

The highest order is generally chosen as $n_{0}=1$ or $n_{0}=2$, where the latter effects a spatial concentration of loads in the dipole axis. Furthermore, $s=1$ stresses the spatial concentration of loads around the dipole.

In the direct potential approach in combination with the blurred dipole, the total potential $\Phi(x) \approx \Phi_{h}(x)=$ $\sum_{j=1}^{N} \varphi_{j}(x) u_{j}$ is projected into the FE space and, using variational and FE techniques for (1), a linear system

$$
K \underline{u}=\underline{J}^{\text {blur }}
$$

is derived with the same stiffness matrix as in (8). The righthand side vector $\underline{J}^{\text {blur }} \in \mathbb{R}^{N}$ has only $C$ nonzero entries at the neighboring $\mathrm{FE}$ nodes to the considered dipole location. It is determined by

$$
\left(\underline{J}^{\text {blur }}\right)^{[i]}= \begin{cases}\underline{j}_{l}^{[c]}, & \text { if } \exists c \in\{1, \ldots, C\}: i=\operatorname{GLOB}(c) \\ 0, & \text { otherwise }\end{cases}
$$

for a source at location $\mathbf{x}_{l}$, where the function GLOB determines the global index $i$ to each of the local indices $c$.

3) Efficient Solution Methods: We employ an algebraic multigrid preconditioned conjugate gradient (AMG-CG) method for solving the linear systems (7) and (14). We solve up to a relative error of $10^{-8}$ in the controllable $K N^{-1} K$-energy norm (with $N^{-1}$ being one V-cycle of the AMG) [22].

As shown above, the linear systems (7) and (14) have the same stiffness matrix (8), but the right-hand side vector is dense for the subtraction approach (9) and sparse with $C$ entries (the number of neighboring FE nodes) for the blurred dipole approach (15). This has implications for the computational effort when using the lead field basis approach [24] (additionally, see [7], [8], [21]), which limits the total number of FE linear equation systems to be solved for any inverse method to the number of sensors nb_sens. After computing the nb_sens vectors of the lead field basis, each forward problem can be solved by a single multiplication of the right-hand side $\underline{J}$ with the basis [24], resulting in a computational effort of $2 *$ nb_sens $* P$ operations, where $P=N$ for the subtraction approach and $P=C$ for the blurred dipole direct potential approach. Note that the lead field basis can not be used when the mesh is adapted according to varying source positions within the inverse problem. We therefore attempt to avoid local mesh refinement techniques as used in [2] and [4].

\section{B. FE Volume Conductor Models}

In source reconstruction, head modeling is generally based on segmented magnetic resonance (MR) data, where curved tissue boundaries have a stair-step representation. We segmented a three tissue realistically-shaped head model with compartments skin, skull and brain and an isotropic voxel size of $1 \mathrm{~mm}^{3}$ from a T1- and proton-density-weighted MR dataset of a healthy 32-year-old male subject. The bi-modal MR approach allowed an improved modeling of the skull-shape as described in detail in [23]. We chose conductivities of $0.33,0.0042$, and $0.33 \mathrm{~S} / \mathrm{m}$ for the three compartments [5].

For node-shift hexahedra evaluation purposes, we furthermore discretized a four-compartment sphere model in a 3-D data volume with $1 \mathrm{~mm}^{3}$ voxel resolution. The layers represent the compartments skin, skull, cerebrospinal fluid and brain with outer surfaces of radii $92,86,80$, and $78 \mathrm{~mm}$, respectively. We chose conductivities of $0.33 \mathrm{~S} / \mathrm{m}, 0.0042,1.0$, and $0.33 \mathrm{~S} / \mathrm{m}$ for the four compartments [2], [19].

\section{Generation of Hexahedral FE Meshes}

Voxels from a segmented MR volume can be used directly as hexahedral elements, possibly reducing resolution by prior subsampling of the volume as we do below for our volume conductor models. In order to increase conformance to the real geometry and to mitigate the stair-case effects of a voxel mesh, a technique was proposed in [6] to shift nodes on material interfaces in order to obtain smoother and more accurate boundaries. Nodes on a two-material interface are moved into the direction of the centroid of the set of incident voxels with minority material, i.e., the material occuring three times or less in the 8 surrounding voxels. If the centroid of these minority voxels relative to a node is $(x, y, z)$, it is shifted by

$$
(\Delta x, \Delta y, \Delta z)=(n s * x, n s * y, n s * z)
$$



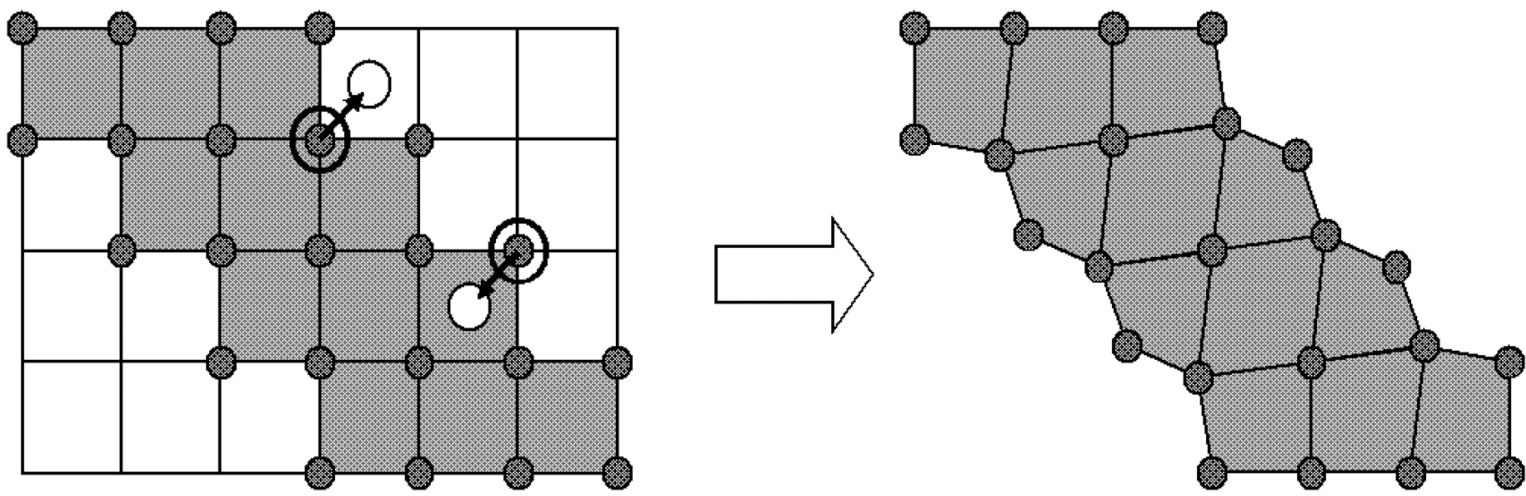

Fig. 1. Concept of the hexahedral node-shift approach for the smoothing of interface boundaries in a 2D scenario: On the left side of the figure, the procedure is illustrated for only two boundary nodes from which one is moved outside and the other one is moved inside towards the centroids of their minority elements. The final result of the node-shift, a smoothed boundary representation using deformed hexahedra, is shown on the right side.

with the user-defined node-shift factor $n s \in[0,0.5)$ (cf. Fig. 1 ). The choice $n s \in[0,0.5)$ ensures that interior angles at element vertices remain convex and the Jacobian determinant remains positive [6].

\section{Error Measures in Sphere Models}

In [15], series expansion formulas were derived for a mathematical dipole in a multilayer sphere model, denoted now as the analytical solution. We compare analytic and numeric solutions using two error criteria that are commonly evaluated in source analysis [2], [14], [19], the relative difference measure (RDM)

$$
\mathrm{RDM}=\sqrt{\sum_{i=1}^{s}\left(\frac{\underline{\phi}_{\text {ana }}^{[i]}}{\left\|\underline{\phi}_{\text {ana }}\right\|_{2}}-\frac{\underline{\phi}_{\text {num }}^{[i]}}{\left\|\underline{\phi}_{\text {num }}\right\|_{2}}\right)^{2}}
$$

and the magnification factor (MAG)

$$
\frac{\mathrm{MAG}=\left\|\underline{\phi}_{\text {num }}\right\|_{2}}{\left\|\underline{\phi}_{\text {ana }}\right\|_{2}}
$$

where $\|\cdot\|_{2}$ denotes the Euclidian norm and $\underline{\phi}_{\text {ana }}, \underline{\phi}_{\text {num }} \in$ $\mathbb{R}^{\text {nb_sens }}$ the analytic or numeric solution vectors at measurement electrodes. The RDM is a measure for the topography error and the MAG indicates changes in the potential amplitude.

We furthermore define the node-shift improvement factor for the RDM (MAG) as the ratio of the RDM (MAG-1) in the regular $(n s=0)$ versus the RDM (MAG-1) in a node-shifted $(n s>0)$ hexahedra model.

\section{E. Parameter Choice for the Blurred Dipole in the Direct Potential Approach}

We choose the parameters of the blurred dipole as follows: The maximal dipole order $n_{0}(10)$ and the scaling reference length $a_{\text {ref }}(11)$ are set to $n_{0}=2$ and $a_{\text {ref }}=20.0 \mathrm{~mm}$, respectively. Since the chosen mesh size (discussed later) is a large factor smaller than the reference length, the second order term $\left(\Delta \bar{x}_{c l}^{r}\right)^{2}$ is small and the model focuses on fulfilling the dipole moments of the zeros and first order. The exponent of the source weighting matrix in (12) is fixed to $s=1$ and the regularization parameter in (13) is chosen as $\lambda=10^{-6}$. The settings effect a spatial concentration of the monopole loads in the dipole axis around the dipole location and gave best results in former

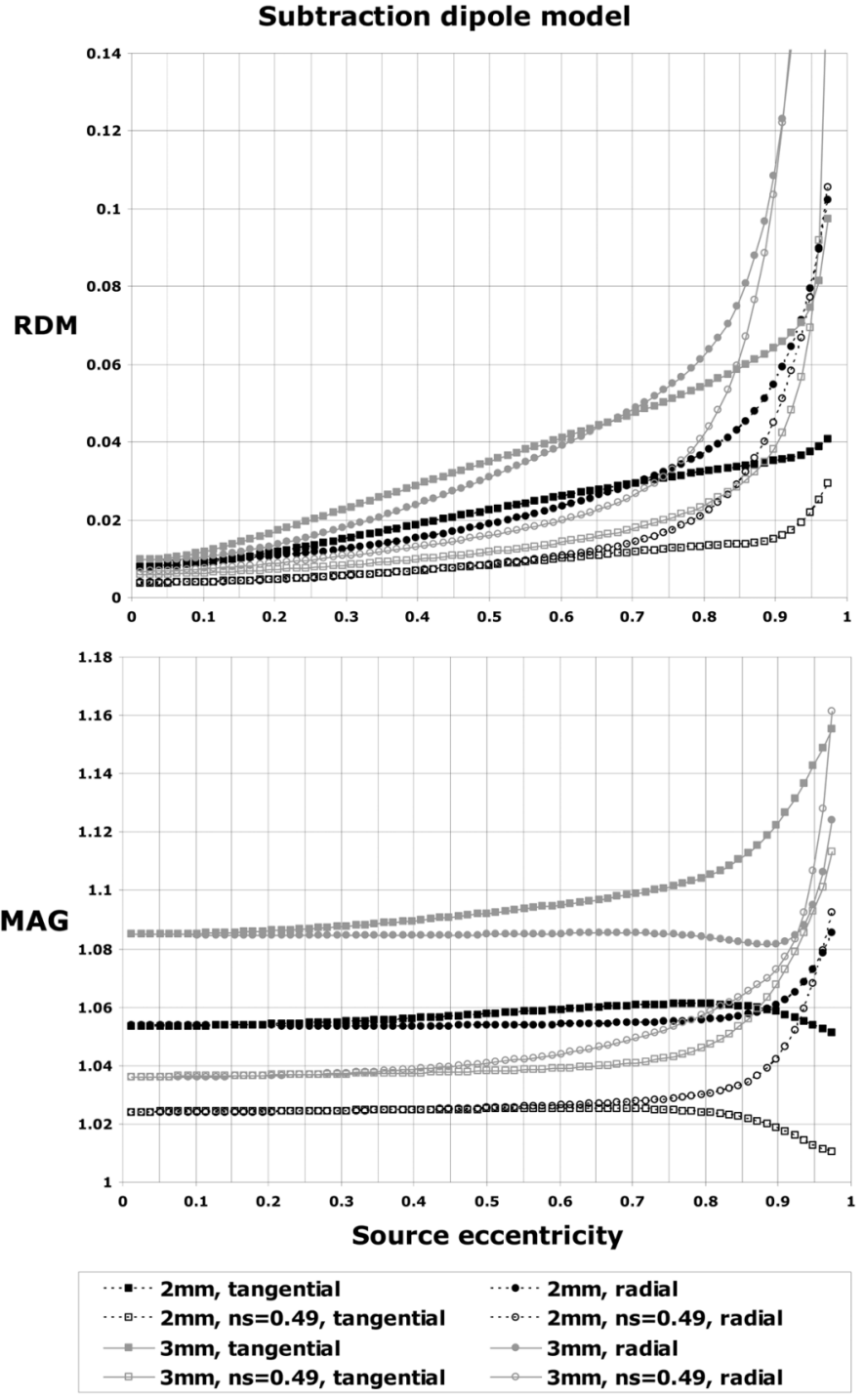

Fig. 2. Subtraction potential approach: Comparison of the numerical accuracy for regular $(n s=0)$ and node-shifted $(n s=0.49) 2$ - and 3-mm hexahedra models for radially and tangentially oriented sources.

evaluations of the presented blurred dipole model in tetrahedra [5], [23] and also regular hexahedra volume conductors [23]. 
TABLE I

SUBTRACTION APPROACH: RDM AND MAG NODE-SHIFT IMPROVEMENT FACTORS FOR 2-mm HEXAHEDRA MODELS

\begin{tabular}{|c|c|c|c|c|c|c|c|}
\hline & \multicolumn{3}{|c|}{ tangential } & \multicolumn{3}{c|}{ radial } \\
\hline \hline & Min & Max & Average & Min & Max & Average \\
\hline \hline$n s$ & \multicolumn{5}{|c|}{ RDM improvement factors } \\
\hline 0.20 & 1.26 & 1.52 & 1.49 & 1.03 & 1.51 & 1.41 \\
\hline 0.40 & 1.38 & 2.29 & 2.12 & 1.00 & 2.19 & 1.90 \\
\hline 0.49 & 1.38 & 2.74 & 2.48 & 0.97 & 2.44 & 2.05 \\
\hline \hline$n s$ & & \multicolumn{7}{|c|}{ MAG improvement factors } \\
\hline 0.20 & 1.40 & 1.62 & 1.43 & 1.00 & 1.41 & 1.35 \\
\hline 0.40 & 1.96 & 3.17 & 2.10 & 0.96 & 1.98 & 1.80 \\
\hline 0.49 & 2.21 & 4.91 & 2.49 & 0.93 & 2.24 & 2.00 \\
\hline
\end{tabular}

\section{RESULTS}

As a programming platform for the presented subtraction and direct potential approach, we used our software environment IP-NeuroFEM [20].

\section{A. Evaluation of the Hexahedra Node-Shift in Sphere Models}

Hexahedral models of the 4-layer sphere were subsampled to 2 - (426 K nodes) and 3-mm ( $130 \mathrm{~K}$ nodes) voxels and node-shift factors $n s(16)$ of 0 (regular), 0.2, 0.4, and 0.49 were used for our evaluation. To achieve independence of the specific choice of the sensor configuration, we distribute nb_sens $=134$ electrodes in a most-regular way over the outer sphere surface. Comparisons between the numeric and the analytic solutions at the electrode positions are carried out for dipoles located on one axis at depths (eccentricities) of $0 \%-97 \%$ (in 1-mm steps) of the inner layer (78-mm radius) using both radial and tangential orientations. We limit the eccentricity to $97 \%$, because it can be expected that the dipole location is at least $2 \mathrm{~mm}$ below the surface of the innermost sphere in the middle of the grey matter compartment. We use dipole strengths of $1 \mathrm{nAm}$.

1) Subtraction Potential Approach: Fig. 2 plots RDM and MAG for the regular $(n s=0)$ and the node-shifted $(n s=0.49)$ 2- and 3-mm hexahedra models for all realistic source eccentricities. In the 2-mm model, we observe a maximal RDM of 0.105 and a maximal MAG of $9.2 \%$ over all depths and for both source orientations. For the 3-mm model, RDM accuracies below 0.14 are only achieved for eccentricities up to $91 \%$ and therefore for the vast majority of realistic source positions, but the results for higher eccentricities are above this threshold and the MAG is equipped with an error of up to $16.1 \%$. In Table I, minimal, maximal and average RDM and MAG node-shift improvement factors are shown for the 2-mm model. For the 3-mm model, the results are very similar (only shown for $n s=0.49$ in Fig. 2). The average improvement factors for both mesh resolutions increase continuously with increasing node-shift values and, for the maximal examined deformation, they are higher than 2.28 for tangential and 1.6 for radial sources. However, as it can be observed in both Fig. 2 and Table I, the node-shift might cause

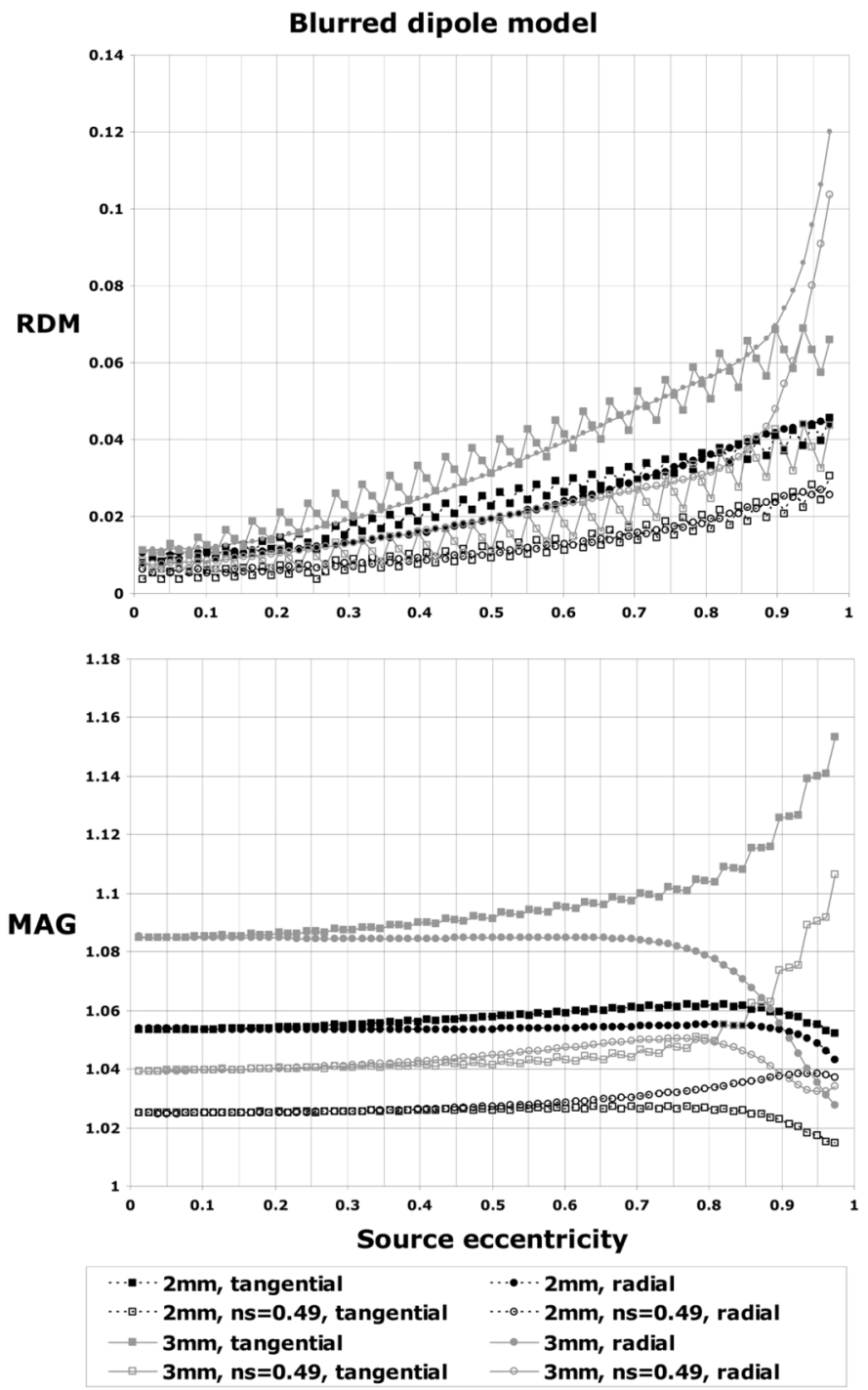

Fig. 3. Direct potential approach: Comparison of the numerical accuracy for regular $(n s=0)$ and node-shifted $(n s=0.49) 2$ - and 3-mm hexahedra models for radially and tangentially oriented sources.

a deterioration of the overall error for sources located within a deformed element or in its direct neighbor element.

2) Direct Potential Approach: In Fig. 3, RDM and MAG are plotted for regular $(n s=0)$ and node-shifted $(n s=0.49) 2$ and 3-mm hexahedra models for all realistic source eccentricities. Again, the error curves are rising with increasing source eccentricity. When compared to the numerical performance of the subtraction approach, the direct approach is less sensitive with smaller errors for sources close to conductivity discontinuities. However, due to variations of the dipole approximation of the blurred dipole model depending on the location within an element, error curve oscillations can be observed. The node-shift improvement factors for the 2-mm model are shown in Table II. All factors are above 1.0, so that a general improvement through node-shifting can be concluded. We achieve very similar results for the 3-mm model, the only significant difference to the $2-\mathrm{mm}$ results is that the MAG improvement factors for the two most eccentric radial sources is slightly below one (see Fig. 3). The average improvement factors for both mesh resolutions increase 
TABLE II

DIRECT APPROACH: RDM AND MAG NODE-SHIFT IMPROVEMENT FACTORS FOR 2-mm HEXAHEDRA MODELS

\begin{tabular}{|c|c|c|c|c|c|c|c|}
\hline & \multicolumn{3}{|c|}{ tangential } & \multicolumn{3}{c|}{ radial } \\
\hline \hline & Min & Max & Average & Min & Max & Average \\
\hline \hline$n s$ & \multicolumn{5}{|c|}{ RDM improvement factors } \\
\hline 0.20 & 1.24 & 1.65 & 1.41 & 1.31 & 1.41 & 1.37 \\
\hline 0.40 & 1.43 & 2.57 & 1.88 & 1.62 & 1.83 & 1.73 \\
\hline 0.49 & 1.49 & 3.04 & 2.10 & 1.67 & 1.95 & 1.83 \\
\hline \hline$n s$ & & MAG improvement factors \\
\hline 0.20 & 1.39 & 1.56 & 1.41 & 1.14 & 1.40 & 1.34 \\
\hline 0.40 & 1.91 & 2.68 & 2.00 & 1.18 & 1.93 & 1.74 \\
\hline 0.49 & 2.14 & 3.55 & 2.30 & 1.17 & 2.17 & 1.90 \\
\hline
\end{tabular}

continuously with increasing node-shift values and, for the maximal deformation, they are higher than 2.05 for tangential and 1.56 for radial sources.

\section{B. Application of Node-Shift Hexahedra Meshing to Realistic Volume Conductor Modeling}

The three-compartment realistic volume conductor model was meshed using $2-\mathrm{mm}$ regular and node-shift $(n s=0.49)$ hexahedra. This resulted in hexahedra FE models with $386 \mathrm{~K}$ nodes and $366 \mathrm{~K}$ elements. The dipole strengths are $100 \mathrm{nAm}$.

The potential distribution in the regular and node-shifted hexahedra models were then computed and visualized using both the subtraction (Fig. 4) and the direct potential approach using the blurred dipole (Fig. 5) for a radially and a tangentially oriented source in somatosensory cortex. As it can be observed in the figures, with regard to the mesh properties, the three surfaces skin, outer and inner skull are represented in a much smoother way in the node-shifted mesh compared to the stair-step approximation in the regular hexahedra model. While the surfaces of outer and inner skull are directly visible in the node-shift hexahedra model, they otherwise can only be estimated indirectly from the bends in the isopotential lines at both skull surfaces. The consequence with regard to the field patterns is, that the smoothness property of the mesh is taken over to the isopotential-lines, which at both skull surfaces appear smoother in the node-shifted meshes.

\section{DISCUSSION AND CONCLUSION}

The focus of our study is the validation of a node-shift hexahedral meshing approach for a subtraction and a direct potential approach in FE-based EEG source analysis, a method which was shown to perform well in a biomechanical FE application [6]. The node-shifted hexahedra better describe the smooth tissue boundaries, but, following convergence proofs in FE numerical analysis, they might also cause larger numerical errors.

We chose a four compartment sphere model with the classic conductivity values of $0.33,0.0042,1.0$, and $0.33 \mathrm{~S} / \mathrm{m}$ (see, e.g.,
[2] and [19]), i.e., a ratio of 1:80 between the skull and the brain compartment. Recent works suggest that the skull conductivity should be only 15 [17] to 25 [10] times lower than the brain conductivity. However, in [11], we presented a low resolution conductivity estimation algorithm that we recently applied to the estimation of the brain:skull conductivity, where we found the classic ratio of 1:80 [12]. In any case, when applying the nodeshift hexahedral meshing approach to a four layer sphere model with a skull to brain ratio of 1:15, the results are very similar to the results shown in this paper, the overall numerical errors of both presented numerical approaches are only lower.

From the evaluation in this paper we can conclude that, with average node-shift improvement factors around 2 for a $2-\mathrm{mm}$ hexahedra resolution, both topography and magnitude errors at surface measurement locations are strongly reduced by the node-shift approach, if the source is not located within a deformed element or its direct neighbor. For a 2-mm mesh resolution, the node-shift always improved the results for the direct potential method, while for sources within the deformed element or its direct neighbor, results of the subtraction approach were slightly spoiled for radial sources. With regard to realistic head modeling, we conclude that the boundaries of the skin, outer and inner skull should be smoothed using the hexahedra node-shift, while we would not recommend deforming elements at the grey and white matter surfaces.

For the used zero-mean EEG data, the RDM can be related to the correlation coefficient (CC) through $\mathrm{RDM}=\sqrt{2(1-\mathrm{CC})}$ [19] and a CC above 0.99 (i.e., RDM below 0.14) was associated with a localization error of no more than $1 \mathrm{~mm}$ [9], [19]. We can therefore conclude that, for the presented sphere model and for both the direct and the subtraction approach, regular and especially node-shifted 2 -mm hexahedra models achieve satisfying numerical accuracy. No mesh adaptation is needed in contrast to tetrahedral local mesh-refinement strategies [2], [4], where elements are refined depending on the varying source position within the inverse problem. We can therefore exploit lead field bases [24], the computationally very efficient solution strategies for the EEG and magnetoencephalography (MEG) inverse problem as described in Section II-A. With increasing eccentricity, the errors begin to rise, a behavior, which has also been observed in [1], [2], [4], [5], [14], and [19]. The decrease in numerical accuracy with increasing eccentricity is stronger for the presented subtraction approach compared to the presented direct method. For the direct potential approach, due to the mesh-dependent implementation of the blurred dipole, we observe oscillations in the error curves. This can be explained by the choice of the $C$ neighboring nodes to the source position $\mathrm{x}_{l}$ in formula (10). In our implementation, the $C$ FE nodes are chosen like follows: First, the closest FE node $\mathbf{x}_{p}$ to $\mathbf{x}_{l}$ is determined. For the modeling of the blurred dipole, we then compute monopole sources on those $C$ FE nodes, which have a common edge with $\mathbf{x}_{p}$. As Fig. 3 shows, the best approximation to the mathematical dipole can thus be achieved if the distance $\left|\mathbf{x}_{l}-\mathbf{x}_{p}\right|$ is zero (the source is positioned at a FE-node), while the approximation is worst if $\mathbf{x}_{l}$ approaches the center of an element. With regard to continuous dipole fits during an inverse EEG analysis, this might be a disadvantage of the presented direct approach compared to the presented subtraction method, where error curves and thus inverse cost functions are smooth. 


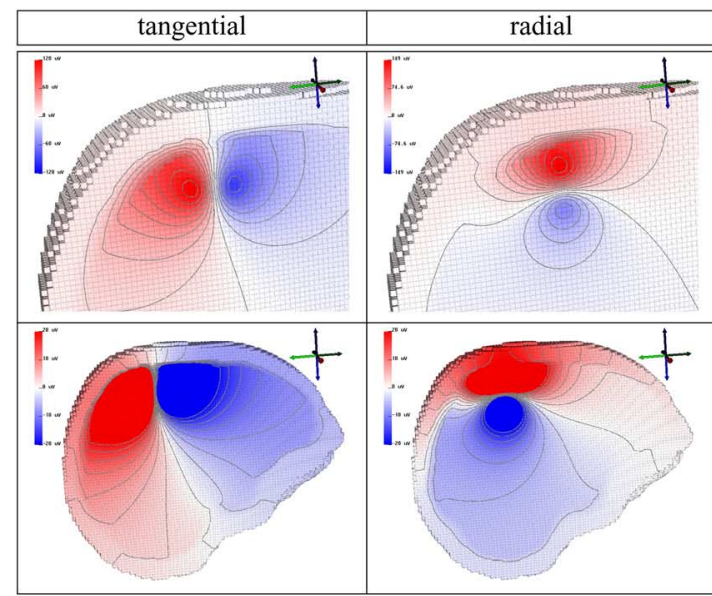

(a)

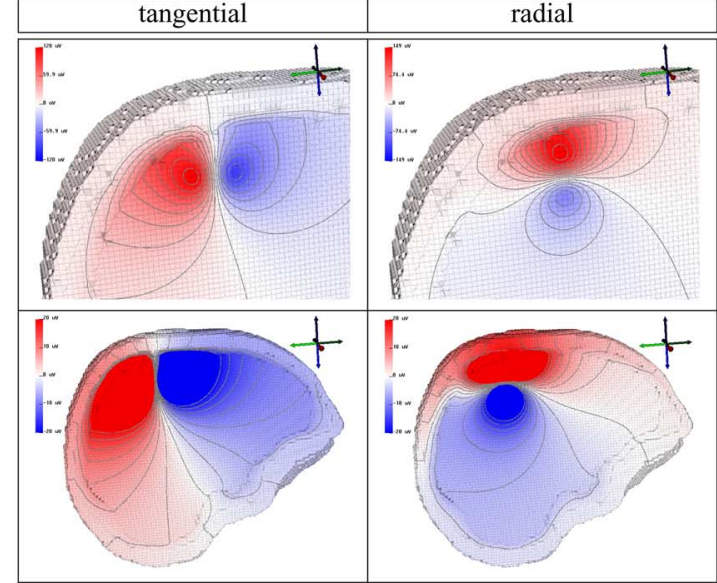

(b)

Fig. 4. Subtraction potential approach in 3-compartment realistic volume conductor of the human head: Visualization of the total potential for a tangentially and a radially oriented dipole in the somatosensory cortex in a regular (a) and a node-shifted ( $n s=0.49$ ) hexahedra FE model (b). The sagittal cutplane was chosen in a distance of $9 \mathrm{~mm}$ from the source position. 15 isopotential lines are shown from the minimal to the maximal potential value in the given plane (upper row) and for an interval of $-20 \mu \mathrm{V}$ to $20 \mu \mathrm{V}$ (lower row). Visualization was carried out using BioPSE [3]. (For interpretation of the references to colour in this figure legend, the reader is referred to the web version of this paper).

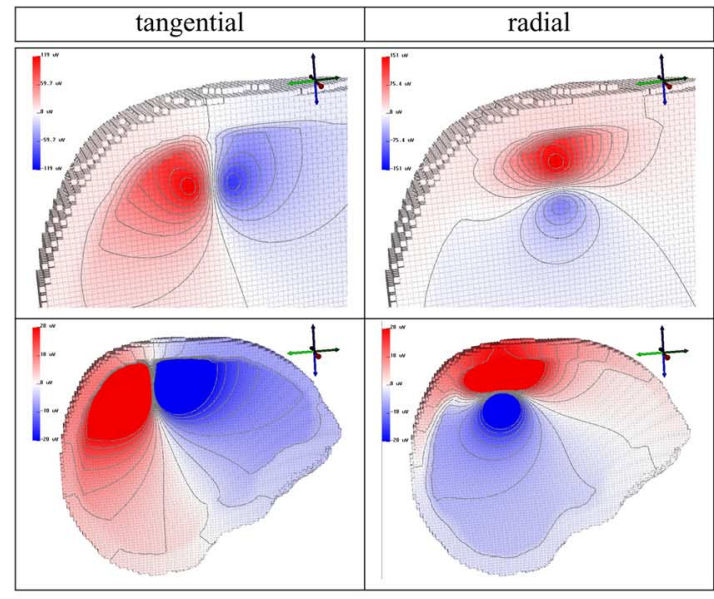

(a)

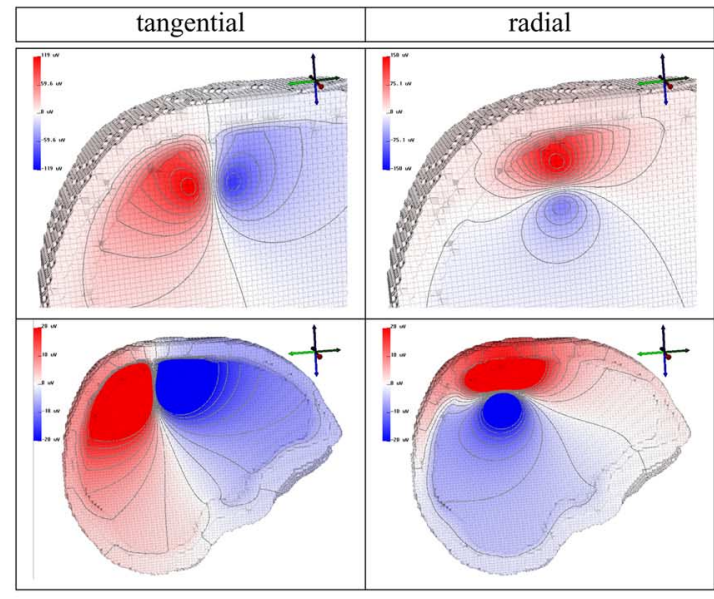

(b)

Fig. 5. Direct potential approach using the blurred dipole in a 3-compartment realistic volume conductor of the human head: Visualization of the potential distribution for a tangentially and a radially oriented dipole in the somatosensory cortex in a regular (a) and a node-shifted $(n s=0.49) 2 \mathrm{~mm}$ hexahedra FE model (b). The sagittal cutplane was chosen in a distance of $9 \mathrm{~mm}$ from the source position. 15 isopotential lines are shown from the minimal to the maximal potential value in the given plane (upper row) and for an interval of -20 to $20 \mu \mathrm{V}$ (lower row). Visualization was carried out using BioPSE [3]. (For interpretation of the references to colour in this figure legend, the reader is referred to the web version of this paper).

\section{ACKNOWLEDGMENT}

The authors would like to thank J. de Munck for providing his software for the analytical solution and for his quick responses whenever needed.

\section{REFERENCES}

[1] K. A. Awada, D. R. Jackson, J. T. Williams, D. R. Wilton, S. B. Baumann, and A. C. Papanicolaou, "Computational aspects of finite element modeling in EEG source localization," IEEE Trans. Biomed. Eng., vol. 44, no. 8, pp. 736-751, Aug. 1997.

[2] O. Bertrand, M. Thévenet, and F. Perrin, J. Nenonen, H.M. Rajala, and T. Katila, Eds., "3D finite element method in brain electrical activity studies," in Biomagn. Localization 3D Modelling, 1991, pp. 154-171.

[3] Problem Solving Environment for Modeling, Simulation, and Visualization of Bioelectric Fields BioPSE, Scientific Computing and Imaging Institute (SCI), 2002 [Online]. Available: http://software.sci.utah.edu/biopse.html
[4] S. P. van den Broek, Volume Conduction Effects in EEG and MEG. Enschede, The Netherlands: Proefschrift Univ. Twente, 1997.

[5] H. Buchner, G. Knoll, M. Fuchs, A. Rienäcker, R. Beckmann, M. Wagner, J. Silny, and J. Pesch, "Inverse localization of electric dipole current sources in finite element models of the human head," Electroencephalogr. Clin. Neurophysiol., vol. 102, pp. 267-278, 1997.

[6] D. Camacho, R. Hopper, G. Lin, and B. Myers, "An improved method for finite element mesh generation of geometrically complex structures with application to the skullbase," J. Biomech., vol. 30, no. 10, pp. 1067-1070, 1997.

[7] N. G. Gencer and C. E. Acar, "Sensitivity of EEG and MEG measurements to tissue conductivity," Phys. Med. Biol., vol. 49, pp. 701-717, 2004.

[8] H. Hallez, B. Vanrumste, P. Van Hese, Y. D'Asseler, I. Lemahieu, and R. Van de Walle, "A finite difference method with reciprocity used to incorporate anisotropy in electroencephalogram dipole source localization," Phys. Med. Biol., vol. 50, pp. 3787-3806, 2005

[9] J. Haueisen, A. Böttner, H. Nowak, H. Brauer, and C. Weiller, "The influence of conductivity changes in boundary element compartments on the forward and inverse problem in EEG and MEG," Biomedizinische Technik, vol. 44, no. 6, pp. 150-157, 1999. 
[10] Y. Lai, W. van Drongelen, L. Ding, K. E. Hecox, V. L. Towle, D. M. Frim, and B. He, "Estimation of in vivo human brain-to-skull conductivity ratio from simultaneous extra- and intra-cranial electrical potential recordings," Clin. Neurophysiol., vol. 116, pp. 456-465, 2005.

[11] S. Lew, C. Wolters, A. Anwander, S. Makeig, and R. S. MacLeod, D. Cheyne, B. Ross, G. Stroink, and H. Weinberg, Eds., "Low resolution conductivity estimation to improve source localization," in Proc. 15th Int. Conf. Biomagnetism International Conference Series, 2007, vol. 1300c, pp. 149-152.

[12] S. Lew, C. Wolters, A. Anwander, S. Makeig, and R. S. MacLeod, "Low resolution conductivity estimation to improve source localization," NeuroImage, in review, 2007.

[13] A. K. Louis, Inverse und schlecht gestellte Probleme. Leipzig, Germany: Teubner-Verlag, 1989.

[14] G. Marin, C. Guerin, S. Baillet, L. Garnero, and G. Meunier, "Influence of skull anisotropy for the forward and inverse problem in EEG: Simulation studies using the FEM on realistic head models," Hum. Brain Mapp., vol. 6, pp. 250-269, 1998.

[15] J. C. de Munck and M. Peters, "A fast method to compute the potential in the multisphere model," IEEE Trans. Biomed. Eng., vol. 40, no. 11, pp. 1166-1174, Nov. 1993

[16] W. Nolting, Grundkurs: Theoretische Physik, Elektrodynamik. Ulmen, Germany: Zimmermann-Neufang, 1992.

[17] T. F. Oostendorp, J. Delbeke, and D. F. Stegeman, "The conductivity of the human skull: Results of in vivo and in vitro measurements," IEEE Trans. Biomed. Eng., vol. 47, no. 11, pp. 1487-1492, Nov. 2000.

[18] J. Sarvas, "Basic mathematical and electromagnetic concepts of the biomagnetic inverse problem," Phys. Med. Biol., vol. 32, no. 1, pp. $11-22,1987$.

[19] P. H. Schimpf, C. R. Ramon, and J. Haueisen, "Dipole models for the EEG and MEG," IEEE Trans. Biomed. Eng., vol. 49, no. 5, pp. 409-418, May 2002.

[20] SimBio: A Generic Environment for Bio-Numerical Simulation, ISTProgram of the European Commission Project No. 10378, 2000-2003 [Online]. Available: http://www.simbio.de

[21] D. Weinstein, L. Zhukov, and C. Johnson, "Lead-field bases for electroencephalography source imaging," Ann. Biomed. Eng., vol. 28, no. 9, pp. 1059-1066, 2000.

[22] C. H. Wolters, M. Kuhn, A. Anwander, and S. Reitzinger, "A parallel algebraic multigrid solver for finite element method based source localization in the human brain," Comp. Vis. Sci., vol. 5, no. 3, pp. 165-177, 2002.

[23] C. H. Wolters, "Influence of tissue conductivity inhomogeneity and anisotropy on EEG/MEG based source localization in the human brain" Ph.D. dissertation, Leipzig, Univ., Leipzig, Germany, 2003 [Online]. Available: http://dol.uni-leipzig.de

[24] C. H. Wolters, L. Grasedyck, and W. Hackbusch, "Efficient computation of lead field bases and influence matrix for the FEM-based EEG and MEG inverse problem," Inverse Prob., vol. 20, no. 4, pp. 1099-1116, 2004.

[25] C. H. Wolters, A. Anwander, D. Weinstein, M. Koch, X. Tricoche, and R. MacLeod, "Influence of tissue conductivity anisotropy on EEG/MEG field and return current computation in a realistic head model: A simulation and visualization study using high-resolution finite element modeling," NeuroImage, vol. 30, no. 3, pp. 813-826, 2006.

[26] Y. Yan, P. L. Nunez, and R. T. Hart, "Finite-element model of the human head: Scalp potentials due to dipole sources," Med. Biol. Eng. Comput., vol. 29, pp. 475-481, 1991.

[27] Y. Zhang, L. Ding, W. van Drongelen, K. Hecox, D. Frim, and B. He, "A cortical potential imaging study from simultaneous extra- and intracranial electrical recordings by means of the finite element method," NeuroImage, vol. 31, pp. 1513-1524, 2006.

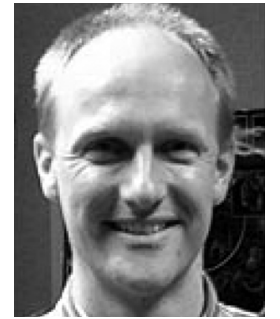

Carsten H. Wolters received the M.Sc. degree (with highest honors) in mathematics with a minor in medicine from the RWTH Aachen, Aachen, Germany, and the Ph.D. (magna cum laude) degree in mathematics from the University of Leipzig, Leipzig, Germany, in 1997 and 2003, respectively.

From 1997 to 2004, he was with the Max Planck Institutes for Human Cognitive and Brain Sciences and Mathematics in the Sciences, Leipzig, and contributed to the EU-project SimBio. In 2004, he joined the Scientific Computing and Imaging Institute at the University of Utah, Salt Lake City. Since 2005, he is a Research Associate with the Institute for Biomagnetism and Biosignalanalysis at the University of Münster, Münster, Germany. His research interests are in the field of neuroscience with a focus on EEG/MEG source analysis.

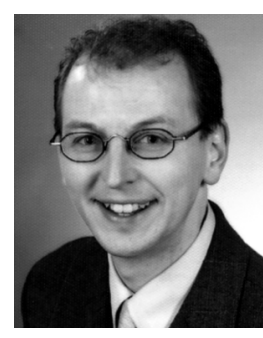

Alfred Anwander received the M.Sc. degree in electrical engineering from the University of Karlsruhe, Karlsruhe, Germany, in 1996, and the M.Sc. (DEA) and $\mathrm{Ph} . \mathrm{D}$. degrees from INSA Lyon Scientific and Technical University of Lyon, Lyon, France, in 1996 and 2001, respectively.

Since 2000, he is a Scientist the Max Planck Institute for Human Cognitive and Brain Sciences, Leipzig, Germany. His research interests are in EEG/MEG source localization and quantitative connectivity from diffusion MRI.

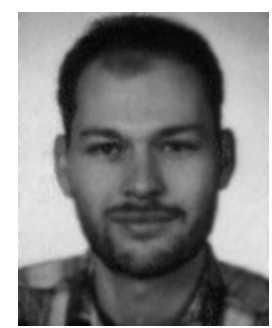

Guntram Berti received the M.Sc. degree (Diplom) in mathematics from the University of Dortmund, Dortmund, Germany, in 1994 and the Ph.D. degree in mathematics from the University of Cottbus, Cottbus, Germany, in 2000

In 2001, he joined the C\&C Research Laboratories of NEC Europe Ltd., St. Augustin, Germany, where he leads the Medical and Life Science Applications Team. He contributed to the EU projects SimBio, GEMSS and @ neurIST. His research interests include mesh generation and geometry processing

for medical applications.

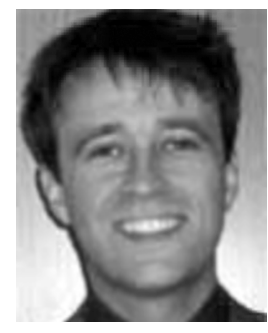

Ulrich Hartmann studied physics in Heidelberg and Göttingen, Germany. The main focus of his studies was in medical physics in the area of SPECT imaging. After having received the diploma degree from the physics department of the University of Göttingen in 1995 he began working towards the $\mathrm{Ph} . \mathrm{D}$. degree at the Max Planck Institute for Human Cognitive and Brain Sciences, Leipzig, Germany, in the field of finite-element modelling.

In 1998, he took the position as a Research Staff Member at the NEC Research Laboratories, St. Augustin, Germany. He continued his work on finite-element models in medicine and started working on the European project SimBio. In 2001, he became Professor of Medical Engineering and Informatics at the University of Applied Sciences, Koblenz, Germany, where he is responsible for the biomechanics laboratory. 